\title{
A REVIEW: DEVELOPMENT OF PESTICIDE SPRAYING MACHINE
}

\author{
Akhilesh K.Bhatkar ${ }^{1}$, P. B. Khope ${ }^{2}$, P. S. Chaudhari ${ }^{3}$ \\ ${ }^{1}$ M.Tech Student, Department of Mechanical Engineering, PCOE, Nagpur-440019 \\ akhileshbhatkar@gmail.com \\ ${ }^{2}$ Assistant Professor, Department of Mechanical Engineering, PCOE, Nagpur-440019 \\ khopepb@yahoo.co.in \\ ${ }^{3}$ Assistant Professor, Department of Mechanical Engineering, PCOE, Nagpur-440019
}

\begin{abstract}
Agriculture has been the backbone of Indian economy. It has to support 17 percent of world population with only 2.3 percent of world's geographical area, 4.2 percent of world's water resource, with 2 percent total consumption of world's total pesticide. To fulfill the need of food modernization of agricultural sector is important. There are many areas in agricultural sector where speed of modernization is quiet slow. One of the main sectors is pesticide spraying machine. By modernization in this sector pesticides can be evenly distributed on farms which will reduce wastage of pesticide and hence prevents wastage of inputs applied on farms which reduces cost of production. Mechanization in pesticide spraying technology will lead to higher productivity with minimum input. Indian farmers are using some traditional spraying mechanisms which lead to wastage of pesticides with some hazardous ill effects on their health. Now day's some technologies are developed and some inventions are there in this field but they are not much suitable for Indian farming conditions.
\end{abstract}

Keywords: Agricultural Sector, Indian Farming, Pesticide Spraying Machine, Mechanization, Modernization, Inventions.

\section{INTRODUCTION}

Farming is called as backbone of Indian economy. Farming is very complicated as it contains various Operations like weeding, sowing, harvesting etc. Apart from this it has to depend on nature for various reasons like climatic changes. Because as climate changes it leads to change in growing conditions of plant in farms. These changes bring various diseases on plants which decreases productivity hence increases cost of production. Hence spraying operation to protect plants from diseases plays an important role in agriculture sector.

In last 50 years farming has undergone great evolution in spraying mechanism to control various diseases on plants. During initial day's farmers was performing hand operated spraying. Later there has been development of various methods to spray out fertilizers and pesticides. But with every development hopes of the farmers to increase productivity with minimum production cost increases tremendously. To fulfill these requirements variousresearches has been performed to limit wastage of pesticide and fertilizers as well as in mechanism of spraying machines.

To understand various problems faced by farmers in India about pesticide spraying machine, this paper presents a review on various mechanisms developed in pesticide spraying machine. Also it highlights some of 'Jugaad' or innovations developed in India. Now a day's these jugaad are getting importance as of innovations.

\section{PESTICIDE SPRAYING MACHINE}

Spraying techniques are commonly divided as high volume $(\mathrm{HV})$, low volume (LV), and ultra low volume (UVL), according to total volume of liquid mixture applied per unit of ground area. And according to spraying techniques pesticide spraying machines are developed to get required output.

\subsection{Hand Sprayer}

The hand sprayer is light weight low capacity pneumatic sprayer made of chromium plated brass tank. Basic principal of operation of this pump is has done by air pump which remains inside the tank. The sprayer has short delivery tube to which adjustable nozzle is attached. For spraying tank is usually filled to about $70 \%$ of its capacity and pressurized by air pump. This compressed air causes mixture of pesticide to force out on operation of trigger. It is commonly used for small nurseries, small or household gardens.

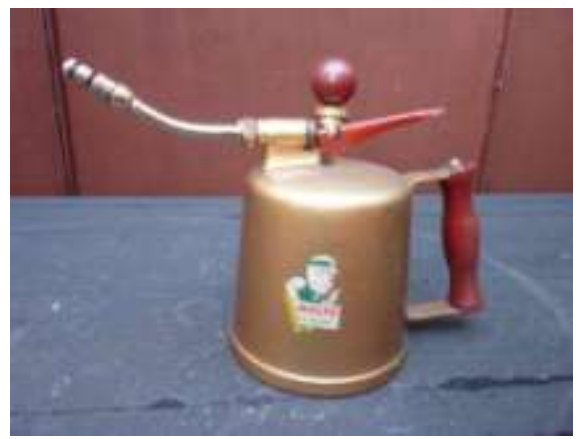

Fig.1. Hand sprayer 


\section{Hand Sprayer Specifications}

Capacity: 0.5-3 liters

Diameter of tank: $130 \mathrm{~mm}$

Height: $210 \mathrm{~mm}$

Weight: $1.2 \mathrm{~kg}$

\subsection{Knapsack Sprayer}

Knapsack sprayer is medium capacity pump which consists of a pump and air chamber permanently installed in a 9 to 22.5 liters tank. This sprayer has harness that allows it to be carried on operators back. This pump is usually activated by moving a lever. The handle of pump is extended over the shoulder so as to make it possible for operator to pump with one hand and spray with other hand. Uniform pressure is maintained by continuous operation of pumping. It is commonly used for spraying insecticides and pesticides on small trees, shrubs and row crops. This is the most commonly used pump in India.

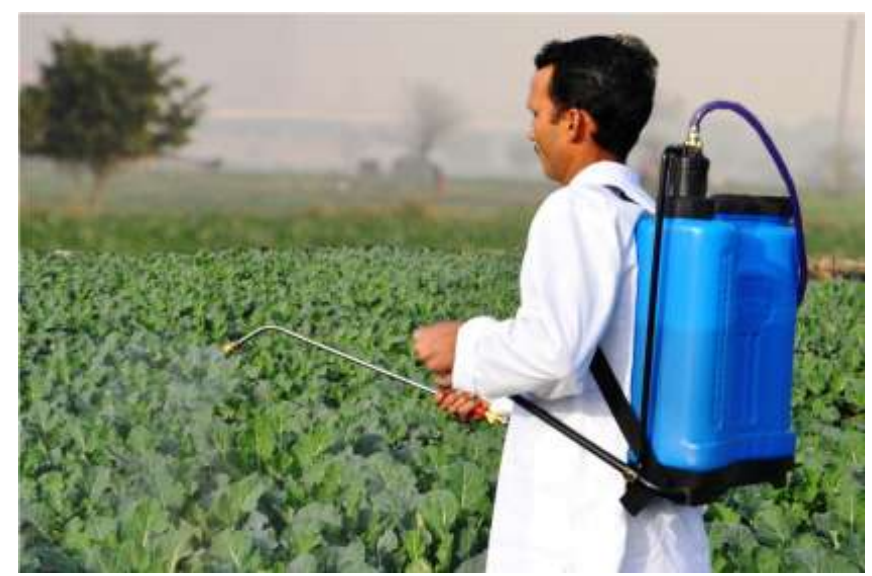

Fig.2. Knapsack sprayer

\section{Knapsack sprayer specifications:}

Capacity: 9-22.5 liters

Power required: One person

Pump cylinder inner diameter: $39-42 \mathrm{~mm}$

Displacement volume: $85-90 \mathrm{ml}$

\subsection{Tractor Mounted Sprayer}

Tractor mounted sprayers are high capacity hydraulic energy sprayers which utilizes the power of tractor to operate pump. The complete spraying assembly is mounted on three point linkages of tractor. Spraying of pesticides is carried out through maximum of 20 nozzles depending upon make of sprayer and formation of crops. These nozzles are carried by spray boom which is adjustable depending upon height of plants. The overhead spray boom is useful for tall crops while ground spray boom is designed for small to medium heighted crops. This sprayer assembly consists of fiber glass or plastic tank, pump assembly, suction and discharge pipes with filters, pressure gauges and regulators, spray boom which all are designed for high pressure, high discharge conditions.

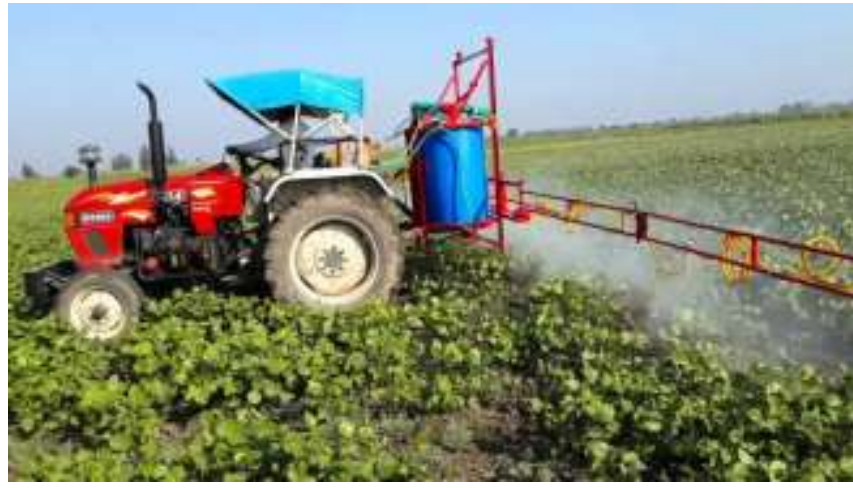

Fig.3. Tractor mounted sprayer

\section{Tractor mounted sprayer specifications:}

Capacity: 200-400 liters

Power required: Power of tractor

Weight of assembly: $150 \mathrm{~kg}$

Overall length: $6340 \mathrm{~mm}$

Overall width: $1290 \mathrm{~mm}$

Overall height: $1570 \mathrm{~mm}$

Field capacity: 8 ha/day (with 14 nozzles)

\subsection{Aerial Sprayer}

An aerial sprayer is high capacity sprayer which is beneficial for farmers having large farms. It uses modern technique in agricultural field. In this spraying is done with the help of small helicopter controlled by remote. Tank and spraying boom is attached to helicopter using various techniques. Spraying boom carries variable number of nozzles and spraying is worked out on farm from some altitude. This is rarely used in India as it is very costly.

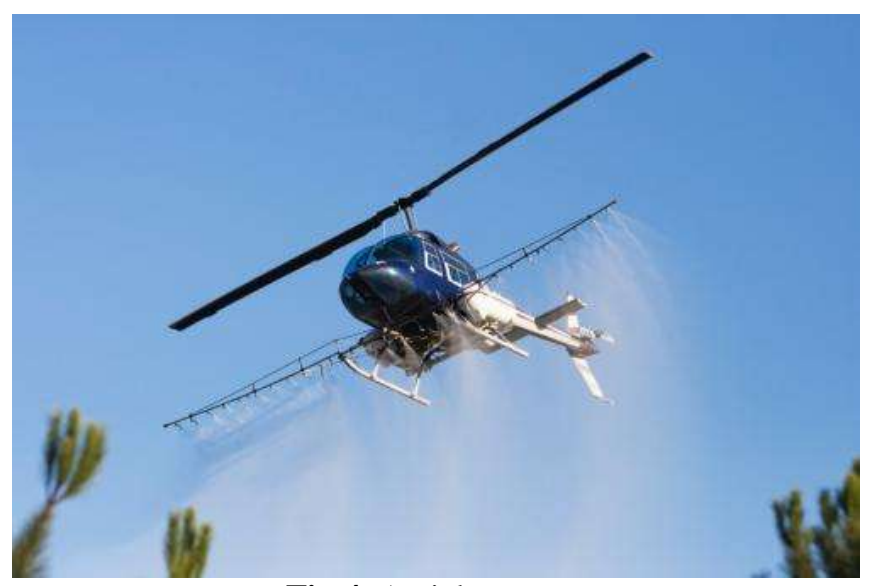

Fig.4. Aerial sprayer

\subsection{Bicycle Mounted Sprayer}

Bicycle mounted sprayers is medium capacity innovative pesticide sprayers which utilizes the power of bicycle to operate pump. This is developed by Mansukhbhai Jagani, a Gujarat based farmer who is famous for his agricultural 'Jugaad'. He simply made an assembly of modified sprocket-pump, tank, and an adjustable sprayer. This portable system is mounted on bicycle with drum carrying pesticide firmly attached to frame of bicycle. The 
reciprocating pistons of pump are connected to sprocket with chain linkage. Output of this pump is given to boom carrying number of nozzles. While pulling along the bicycle forward or backward movement of chain and modified sprocket arrangement is transferred to pump assembly which pumps air into tank to generate power for spraying. This pesticide spraying machine is not useful for every condition of agriculture in India.

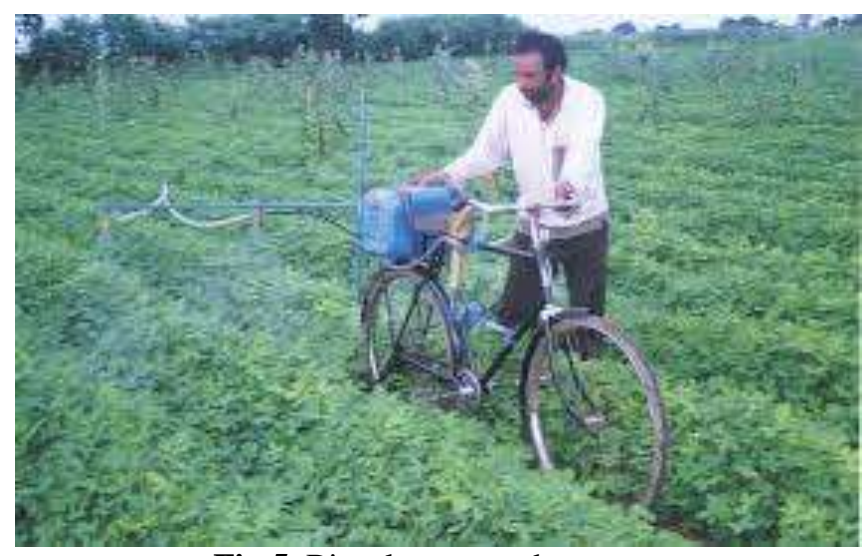

Fig.5. Bicycle mounted sprayer

\section{Bicycle mounted sprayer specifications:}

Capacity: 30 liters

Power required : Power of human

Weight of assembly: Not specified

Field capacity: 1acre/45 minutes

\subsection{Motorcycle mounted sprayer}

Motorcycle mounted sprayers is an innovative pesticide sprayers which utilizes the power of an engine to operate pump. This is developed by Ganeshbhai Nanjibhai Dodiya, a Gujarat based farmer. He just mounted his innovative sprayer on Enfield motorcycle which is powered by engine. The spraying of pesticides is done through a nozzle mounted on a spray boom installed at back of motorcycle. The pesticide filled tank is placed above the pump and gravity ensures the pump gets automatically primed as fluid tends to flow into pump and flood the chamber. As this 'Jugaad' requires Bullet it is not affordable to every farmer in India.

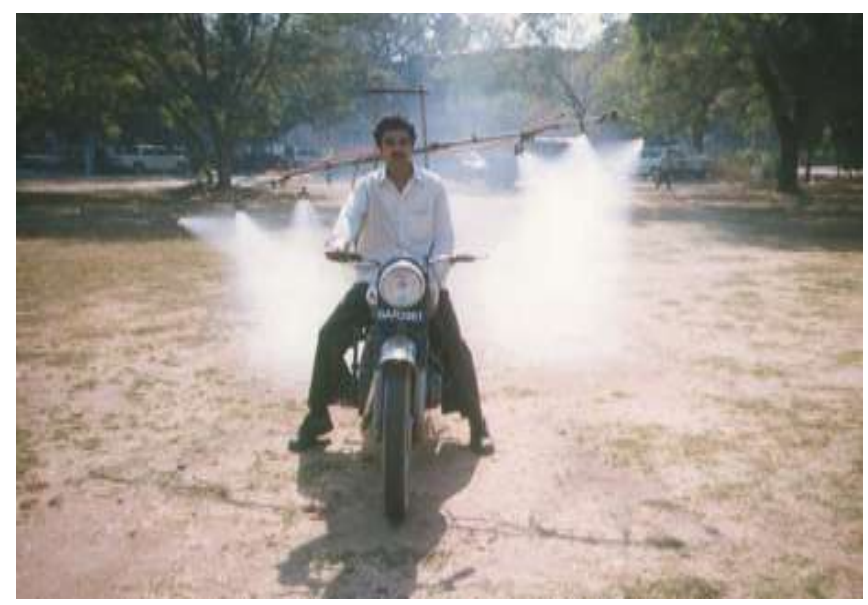

Fig.6. Motorcycle mounted sprayer

\section{Motorcycle mounted sprayer specifications:}

Capacity: 70 liters

Power required: Power of engine $(5 \mathrm{Hp})$

Weight of assembly: Not specified

Field capacity: $1.5 \mathrm{acre} / \mathrm{hr}$

\section{CONCLUSION}

The development of mechanization of pesticide spraying machine is revived. It is concluded that pesticide spraying machine is main factor in India for development of agricultural sector. So to improve productivity there is need of development in mechanized agriculture is needed. By mechanization we can efficiently reduce the effort of labors and uniformly spray the pesticide all over the farm. So there is need of development of user friendly, efficient and low cost mechanization in agricultural sector especially in pesticide spraying machine.

\section{REFERENCES}

[1] Nitin Das, "Agricultural Fertilizers and Pesticides Sprayers - A Review"," IJIRST -International Journal for Innovative Research in Science \& Technology| Volume 1 | Issue 11 | April 2015 ISSN (online): 2349-6010"

[2]Laukik P. Raut, "Design, development and fabrication of agricultural pesticides sprayer with weeder"," International Journal of Applied Research and Studies (iJARS)ISSN: 2278-9480 Volume 2, Issue 11 (Nov - 2013)"

[3]R. Joshua, V. Vasu and P. Vincent "Solar Sprayer AnAgriculture Implement", "International Journal of Sustainable Agriculture 2 (1): 16-19, 2010 ISSN2079-2107" [4]Mahesh M. Bhalerao, "Development and fabrication of smart spray pump"

[5] MM Pandey, "Indian Agriculture - An Introduction", "Fourth Session of the Technical Committee of APCAEM10-12 February 2009, Chiang Rai, Thailand" [6]"State of Indian Agriculture - 2013-2013", "Government of IndiaMinistry of AgricultureDepartment of Agriculture and CooperationDirectorate of Economics and StatisticsNew Delhi"

\section{WEBSITES}

1.http://agricoop.nic.in/dacdivision/machinery1/chap4.pdf

2.http://nif.org.in/innovation/motorcycle_sprayer/244

3.http://nif.org.in/innovation/bicycle_mounted_sprayer/224 